\title{
Colonoscopia de calidad, limpieza y preparación colónica
}

\author{
Alejandra Noble-Lugo* \\ Departamento de Enseñanza e Investigación, Hospital Español de México, Ciudad de México, México
}

En la actualidad hay un claro énfasis en determinar los factores que tienen un impacto positivo en la calidad de la colonoscopia. A continuación se presenta una selección de los trabajos más relevantes presentados en congresos internacionales sobre este tema.

\section{Limpieza y preparación para colonoscopia}

La limpieza intestinal es clave para lograr una colonoscopia de calidad. Un estudio ${ }^{1}$ en 28,725 pacientes (23.3\% con preparación inadecuada), reportó como factores asociados con mala limpieza intestinal la edad avanzada (RM 1.015), sexo masculino (RM 1.35), hospitalización (RM 2.01), estreñimiento (RM 1.37) y rectorragia $(\mathrm{RM}=1.47)$; otro estudio $^{2}$ encontró que sobrepeso $\mathrm{u}$ obesidad y diabetes mellitus son factores de riesgo. Los factores que favorecen una adecuada limpieza intestinal son: apego a las indicaciones de la preparación $(p<0.0001)$, preparación administrada en dosis dividida 0 el mismo día del procedimiento $(p=0.004)$, paciente ambulatorio $(p<0.0001)$, edad $<50$ años $(p<0.0001)$ y menor tiempo entre fin de la preparación e inicio del procedimiento $(p=0.003)^{3}$. Es indispensable lograr una buena preparación porque la limpieza subóptima se asocia con una alta tasa de adenomas no observados previamente $(38.9 \%)$ y adenomas avanzados $(15 \%)$ en la colonoscopia repetida en el siguiente año $0^{4}$.

Dieta: La dieta ha sido muy estudiada por su papel en la preparación intestinal. Un estudio prospectivo, aleatorizado, ciego comparó una dieta baja en residuo vs. dieta sin restricciones y encontró que la dieta sin restricciones en una alternativa que puede lograr del $85 \%$ en pacientes ambulatorios sin factores de riesgo para mala preparación ${ }^{5}$. En caso de optar por dieta baja en residuo, mantener la restricción solo un día es igual de efectivo y mejor tolerado que hacerlo por 3 días $^{6}$.

Tipos de preparación: Existe creciente evidencia de la efectividad y ventajas de la preparación de bajo volumen. Bushyhead, et al. compararon la efectividad de la preparación de bajo volumen (2L PEG con ácido ascórbico), con la de alto volumen (4L PEG) en 1412 colonoscopias; demostraron que ambas lograron adecuada limpieza evaluada por escala de limpieza intestinal de Boston (BBPS), pero la preparación de bajo volumen fue mejor tolerada $(43.6 \%$ vs. $34.4 \%$; $p<0.001)$ y se asoció con mejor experiencia (58\% vs. $51.2 \% ; p<0.009)$, ingesta de más de $90 \%$ de la preparación $(93.3 \%$ vs. $90 \% ; p=0.01)$ y mayor disposición para repetir la preparación $(83.7 \%$ vs. $76.7 \%$; $p<0.001)^{7}$. En el caso de pacientes postoperados de cirugía bariátrica, un estudio demostró que PEG 2L es tan efectivo como 4L; sin embargo la tolerancia y satisfacción es mayor en el grupo de bajo volumen ${ }^{8}$.

En estudios aleatorizados controlados fase $3^{9,10}$ la preparación de muy bajo volumen NER1006 (1L PEG + ácido ascórbico) vs. $2 \mathrm{~L}$ y $4 \mathrm{~L}$ reportó que $1 \mathrm{~L}$ fue la más efectiva y con mejor apego y tolerancia en comparación con $2 \mathrm{~L}$ y 4L ${ }^{9}$; NER1006 también demostró seguridad en pacientes con falla renal leve a moderada ${ }^{10}$.

Edad avanzada: Es un factor de riesgo para inadecuada limpieza. Los factores de asociados con preparación exitosa en este grupo son: buena preparación previa 
(RM 2.29), buena tolerancia a la preparación (RM 1.29), dosis dividida (RM 2.43), dieta baja en fibra por 3 días (RM 2.45) y colonoscopia no más de 5 horas después de la preparación (RM 2.67) ${ }^{11}$. Un estudio de seguridad de NER1006 demostró ser una opción segura en este grupo de pacientes ${ }^{12}$. Para reducir la intolerancia a la ingesta completa de la preparación, Lee, et al. evaluaron el efecto de $15 \mathrm{mg}$ de mosaprida antes de cada dosis de PEG en preparación dividida, demostrando que se asoció con mejor puntaje de BBPS total (8.53 vs. $8.24 ; p=0.033$ ), en colon derecho ( 2.75 vs. $2.61 ; p=0.044$ ) y colon transverso (2.89 vs. $2.79 ; p=0.030)$ en comparación con quienes no recibieron mosaprida, logrando también una reducción en la sensación de llenura gástrica ${ }^{13}$.

Niños: La dosis dividida es más efectiva y mejor tolerada ${ }^{14}$.

Educación: Múltiples estudios ${ }^{15,16}$ demostraron la utilidad de estrategias educativas para el paciente como el envío de recordatorios mediante mensajes de texto automatizados mejoran la calidad de la limpieza intestinal, reduce la tasa de cancelaciones e incrementa la satisfacción de los pacientes, aunque otros estudios no encontraron diferencias significativas. El estudio prospectivo multicéntrico COLOPRAPP demostró que reforzar la educación del paciente mediante una aplicación para celular los 3 días previos al procedimiento mejoró la calidad de la preparación (BBPS 7.6 vs. 6.7; $p<0.0001)$, ADR (35 vs. $28 \%$ ) y la detección de adenomas planos en colon derecho $(p=0.004)$ comparado con el grupo sin reforzamiento ${ }^{17}$.

\section{Colonoscopia de calidad}

Aunque el indicador de calidad más relevante en colonoscopia es la tasa de detección de adenomas (ADR), existen otros indicadores cuya relevancia e impacto han sido analizados. Debido a que el cáncer colorrectal de intervalo (CCRi) es dos veces más frecuente en el colon derecho, Kosinski, et al. determinaron la tasa ADR proximal vs. ADR distal en 295,454 colonoscopias, demostrando que la ADR proximal se incrementa en pacientes $>70$ años comparado con $<40$ años $(p=0.0001)^{18}$. Un análisis retrospectivo de 3356 pacientes reportó ADR, la ADR proximal, la tasa de adenomas avanzados (AvADR), tasa de adenomas sésiles serrados (SDR) y el número de adenomas por colonoscopia (APC) que fueron de 60, 43.2, 16.9, 4.2 y $1.7 \% \pm 2.4$, respectivamente; demostró que la ADR proximal representa al menos $70 \%$ de la ADR global y tiene buena correlación con los demás indicadores ${ }^{19}$. Un estudio multicéntrico prospectivo demostró que la tasa de detección de pólipos (PDR) tiene fuerte correlación con ADR y APC, por lo que pudiera ser considerado un indicador de calidad apropiado ${ }^{20}$.

Retroalimentación de los programas de calidad: Una revisión sistemática y metaanálisis de Calo, et al. (8 estudios; $N=88,108$ colonoscopias) demostró que las intervenciones educativas como parte integral de los programas de calidad se asocian con incremento en ADR (RR 1.33; IC 95\%: 1.25-1.42) y PDR (RR 1.22; IC 95\%: 1.13-1.32), aunque no modifica la tasa de intubación cecal ni el tiempo de retiro del colonoscopio ${ }^{21}$. Otro metaanálisis reportó que el efecto de este beneficio es más marcado en los endoscopistas con ADR bajos 0 medios que en aquellos que tienen ADR altos ${ }^{22}$.

\section{Técnica y accesorios para mejorar detección de lesiones}

Algunos estudios mostraron la utilidad de técnicas, diversos equipos y accesorios que pueden mejorar la detección de lesiones neoplásicas significativas.

Segunda revisión colon derecho: Un estudio internacional multicéntrico, aleatorizado, en 1011 pacientes demostró que esta maniobra incrementa tanto ADR derecho ( $27.1 \%$ vs. $21.6 \%$; $p=0.042$ ) como PDR del lado derecho del colon (34.5\% vs. $25.3 \%$; $p=0.002$ ) y es una estrategia costo-efectiva ya que no requiere entrenamiento, equipo ni accesorios especiales, por lo que debe realizarse en toda colonoscopia de tamizaje o vigilancia ${ }^{23}$.

Inspección retrógrada del colon: La inspección en retroflexión utilizando equipo Pentax Retro-View ${ }^{\circledR}$ posterior a la revisión frontal convencional, aumentó PDR en $10 \%$, ADR en $9 \%$ y el número de adenomas por colonoscopia (de 1.7 a 2.3) comparado con la colonoscopia de visión frontal únicamente ${ }^{24}$.

Intercambio de agua: Leung, et al. presentaron un metaanálisis que demuestra que esta técnica aumentó de manera significativa la detección de pólipos sésiles serrados comparado con aire ambiente o insuflación con $\mathrm{CO}_{2}{ }^{25}$.

ENDOANGEL ${ }^{\circledR}$ : Es un sistema de asistencia endoscópica en tiempo real basado en inteligencia artificial. Un estudio aleatorizado de 779 pacientes en el Hospital Universitario de Wuhan demostró que ENDOANGEL ${ }^{\circledR}$ aumenta ADR (13.56 vs. $21.91 \%$ ) y PDR (37.08\% vs. $51.29 \%)$ comparado con colonoscopia convencional ${ }^{26}$.

Endocuff ${ }^{\circledR}$ : Diversos estudios demuestran que mejora significativamente ADR y PDR en hasta $10 \%$, especialmente en detectores medios y altos ${ }^{27,28}$.

Endoscopia de imagen mejorada: La combinación de ácido acético $5 \%$ y Blue light imaging (BLI) mejora la visibilidad de lesiones sésiles serradas recurrentes ${ }^{29}$. 


\section{Guías clínicas}

La guía clínica del 2018 de American Cancer Society propuso que el tamizaje de cáncer colorrectal inicie a los 45 años en adultos con riesgo promedio. Chalifoux, et al. demostraron que individuos de 45-49 años presentaron ADR $44.2 \%$, adenomas avanzados en $5.8 \%$ predominantemente en colon ascendente (tasas semejantes a los $<50$ años) por lo que realizar tamizaje a partir de 45 años parece justificado ${ }^{30}$.

\section{Conclusiones}

Los procesos de mejora continua para lograr una colonoscopia de calidad hacen imperativo mantenerse informado respecto a la evidencia más actual sobre aquellos aspectos que tienen un impacto positivo sobre la calidad del procedimiento y de sus resultados.

\section{Financiamiento}

Los autores no recibieron patrocinio para llevar a cabo este artículo.

\section{Conflicto de intereses}

La autora declara ser conferencista de Laboratorios Asofarma, Takeda y Menarini.

\section{Bibliografía}

1. Abu Baker F, Mari A, Gal O, et al. A 10-Year Experience with Bowel Preparation: Predictors and Colonoscopy Outcomes of Inadequate Bowel Cleansing. A Large Cohort Study [abstract]. United European Gastroenterol J 2019;7:P0182.

2. Tunashima $H$, Onda T, Sekine K, et al. An Evaluation of Predictive Factors for Bowel Preparation Before Colonoscopy Using the Boston Bowel Preparation Scale [abstract]. United European Gastroenterol J 2019;7:P1193.

3. Amaro P, Agrawal A, Brink L, et al. Factors Associated with Adequate Bowel Preparation: Observations from The European Colonoscopy Quality Investigation Questionnaire [abstract]. United European Gastroenterol J 2019;7:P0199.

4. Baile Maxia S, Medina Prado L, Mangas Sanjuan C, et al. Diagnostic Yield of Early Surveillance After Inadequate Bowel Preparation on Screening Colonoscopy [abstract]. United European Gastroenterol J 2019;7:P0223.

5. Vallejo Senra N, Monteserin-Ron L, López-Díaz J, et al. Free Diet vs Low-Residue Diet in the Quality of Outpatient Colonoscopy: A Prospective, Randomized, Single Blind Clinical Trial [abstract]. United European Gastroenterol J 2019;7:P0894.

6. Machlab S, Brunet E, Martínez-Bauer E, et al. Low Residue Diet During 3 Days Versus 1 Day In Preparation For Colonoscopy For Popultion Screening Of Colorectal Cancer: Noninferiority Randomised Clinical Trial [abstract]. United European Gastroenterol J 2019;7:P0179.

7. Bushyhead DW, Tiritilli A, Dominitz JA. Comparison of Low Versus High Volume Bowel Preparation Efficacy and Tolerability for Colonoscopy: A Quality Improvement Study. Sesión de trabajos orales presentada en: DDW; 2020 mayo 2-5; Chicago, IL. 527.

8. Melendez-Rosado J, Castaneda D, Hussain I, et al. The Impact of a 2 Liter Versus 4 Liter Bowel Preparation in Post-Bariatric Surgery Patients Undergoing Colonoscopy. Sesión de carteles presentada en: DDW; 2020 mayo 2-5; Chicago, IL. Tu1987.
9. Maida MF, Morreale G, Sinagra E, et al. Effectiveness and Tolerability of Very-Low-Volume Preparation for Colonoscopy: A Prospective, Multicenter Observational Study [abstract]. United European Gastroenterol J 2019;7:P0196.

10. Álvarez González MA, Mokashi M, Hassan C. Colon Cleansing Efficacy And Safety Of $1 \mathrm{~L}$ NER1006 In Patients With Mild To Moderate Renal Impairment: Post Hoc Analysis of Randomised Phase 3 Clinical Trials. [abstract]. United European Gastroenterol J 2019;7:P0190.

11. Maida MF, Sinagra E, Morreale G, et al. Factors Affecting the Quality of Bowel Preparation for Colonoscopy in The Elderly: A Retrospective Analysis of A Prospective Cohort [abstract]. United European Gastroenterol J 2019;7:P1591.

12. Maida MF, Sferrazza S, Sinagra E, et al. Safety of NER1006 in the Elderly: A Post-Hoc Analysis of a Prospective, Multicenter Cohort [abstract]. United European Gastroenterol J 2019;7:P1594.

13. Lee J, Oh Kim T, Park J, et al. Efficacy of Mosapride Citrate with Split Dose of PEG Plus Ascorbic Acid for Bowel Preparation in Elderly Patients: A Randomized Controlled Trial. Sesión de carteles presentada en: DDW; 2020 mayo 2-5; Chicago, IL. Tu1093.

14. Tripathi PR, Poddar U. Yachha SK, et al. Efficacy And Tolerability of a Single Dose vs. Split Dose Polyethilene Glycol for Colonic Preparation in Children: A Randomized Control Study [abstract]. United European Gastroenterol J 2019; 7: OP267.

15. Soroudi C, Storage T, Sanford D, et al. Automated Digital Patient Navigation Program Improves Bowel Preparation Quality for Outpatient Colonoscopy. Sesión de carteles presentada en: DDW; 2020 mayo 2-5; Chicago, IL. Tu1973.

16. Lam TYT, Sung JJY, Wu J, et al. WhatsApp Reminder Improves the Quality of Colorectal Cancer Screening. A Single Blinded Randomized Controlled Study [abstract]. United European Gastroenterol J 2019;7 P1608.

17. Walter B, Frank R, Ludwig L, et al. Impact Of Digital Patient Reinforcement On High Quality Colonoscopy Preparation In CRC Screening Results From The Multi-Center Coloprapp-Study [abstract]. United European Gastroenterol J 2019;7:OP339.

18. Kosinski LR, Lieberman DA, Metcalfe L, et al. Proximal Versus Dista Colon ADR: A New Colonoscopy Quality Metric. Sesión de carteles presentada en: DDW; 2020 mayo 2-5; Chicago, IL. Su1793.

19. Desai M, Dasari CS, Nutalapati V, et al. The Importance of Right Colon Adenoma Detection Rate and Its Correlation with Other Quality Metrics. Sesión de carteles presentada en: DDW; 2020 mayo 2-5; Chicago, IL. Mo1636.

20. Vojtechová G, Ngo O, Grega T, et al. Indicators of Adequate Inspection of Bowel Mucosa: Can We Use Only Polyp Detection Rate (PDR) [abstract]. United European Gastroenterol J 2019;7:P1590.

21. Calo NC, González-Moreno El, Bishay K, et al. Educational Interventions Are Associated with Improved Adenoma Detection Rates: A Systematic Review and Meta-Analysis. Sesión de carteles presentada en: DDW; 2020 mayo 2-5; Chicago, IL. Su1035.

22. Bishay K, Calo NC, Scaffidi MA, et al. Endoscopist Feedback Is Associated With Improvements In Colonoscopy Quality Indicators: A Systematic Review And Meta-Analysis. Sesión de carteles presentada en: DDW; 2020 mayo 2-5; Chicago, IL. Su1053.

23. Tang RS, Lee JW, Chang LC, et al. Comparison of Second Forward View Examination and Conventional Single Forward View Examination of the Right Colon on Adenoma Detection and Screening and Surveillance Colonoscopies in Asia-Pacific Countries: A Multicenter Randomized Controlled Study. Sesión de carteles presentada en: DDW; 2020 mayo 2-5; Chicago, IL. Sa1647.

24. Rath T, Vitali F, Klenske E, et al. Retrograde Inspection of the Colon with a Dedicated Retrograde Viewing Endoscope Increases Adenoma Detection Rate [abstract]. United European Gastroenterol J 2019;7:P0911.

25. Leung F, Leung J, IWATERS. Water Exchange (WE) Significantly Increases Detection Rateo f Sessile Serrated Polyps Compared with Air (AI) Or Carbon Dioxide (CO2) Insufflation - Pooled Data Analysis of Three Randomized Controlled Trials [abstract]. United European Gastroenterol J 2019;7:OP268.

26. $\mathrm{Yu} \mathrm{H}, \mathrm{Wu} \mathrm{L}, \mathrm{Hu} \mathrm{S}$. Seeing is believing [abstract]. United European Gastroenterol J 2019;7:OP337.

27. Sawatzki M, Borovicka J, Baumeler S, et al. Feasibility of Ileal Intubation in Colonoscopy with Endocuff: A Prospective Open Label Comparison [abstract]. United European Gastroenterol J 2019;7:P0191.

28. Karsenti D, Tharsis G, Perrot B, et al. Endocuff-Assisted Colonoscopy vs Standard Colonoscopy on Adenoma Detection Rate in Routine Practice: A Cluster-Randomized Crossover Trial On 2058 Patients [abstract]. United European Gastroenterol J 2019;7:OP338.

29. Yamamoto S, Varkey J, Hedenstrom P. Acetic Acid in Combination with Blue Light Imaging: A New Method to Improve the Detection of Recurring Sessile Serrated Lesions in The Colon [abstract]. United European Gastroenterol J 2019;7:OP352.

30. Chalifoux S, Tsai F, Strum W. Outcomes of Colorectal Cancer Screening Starting at the Age of 45 in Average-Risk, Asymptomatic Patients [abstract]. United European Gastroenterol J 2019;7:P1153. 